\title{
Total body potassium depletion and renal tubular dysfunction following carbenoxolone therapy
}

\author{
R. J. DiCKINSON* \\ M.B., M.R.C.P.
}

\author{
R. SWAminathan $\dagger$ \\ M.B. B.S., Ph.D.
}

Departments of Gastroenterology* and Chemical Pathology, $\uparrow$ Leeds General Infirmary, Leeds LS1 3EX

\begin{abstract}
Summary
A patient with hypokalaemia-associated renal tubular damage following carbenoxolone therapy is described. The hypokalaemia was reflected by a low level for total body potassium (TBK) and investigations confirmed renal tubular dysfunction. Hypokalaemic nephropathy may be secondary to intracellular potassium depletion rather than the hypokalaemia itself.
\end{abstract}

\section{Introduction}

A patient is reported who had total body potassium depletion and renal tubular dysfunction secondary to carbenoxolone therapy; a complication that has only been reported once before in a well documented case (Mitchell, 1971). Results of investigations in this patient demonstrate some of the factors involved in the pathogenesis of hypokalaemic nephropathy.

\section{Case report}

A 64-year-old man was started on carbenoxolone sodium (Duogastrone) $50 \mathrm{mg}$ four times/day for duodenal ulcer. Eight weeks later he complained of ankle swelling. Marked peripheral oedema and an elevated blood pressure were noted. The carbenoxolone was stopped and cyclopenthiazide $0.25 \mathrm{mg}$ with potassium chloride $600 \mathrm{mg}$ (Navidrex K)/day and methyldopa $250 \mathrm{mg}$ twice/day were prescribed. Thereafter he noticed polyuria, nocturia and muscle weakness and by the time of his presentation at the General Infirmary at Leeds one week later, he was unable to walk unassisted. Generalized muscle weakness and moderate ankle oedema were noted and his blood pressure was $170 / 90 \mathrm{mmHg}$. Investigations showed: (mmol/l) sodium 150; potassium 1.4; bicarbonate $>48$, urea $3 \cdot 3$. The calculated serum osmolality was $315 \mathrm{mosmol} / \mathrm{l}$. Serum creatinine was $75 \mu \mathrm{mol} / 1$. Serum glutamic oxalo-acetic acid transaminase (SGOT) was 97 i.u./1 (normal <22) and bilirubin was $28 \mu \mathrm{mol} / \mathrm{l}$. Urinary output on the second hospital day was $2975 \mathrm{ml}$ (Fig. 1). The urine revealed a trace of albumin only and the urinary electrolytes were $(\mathrm{mmol} / 24 \mathrm{hr})$ : urea 345 ;

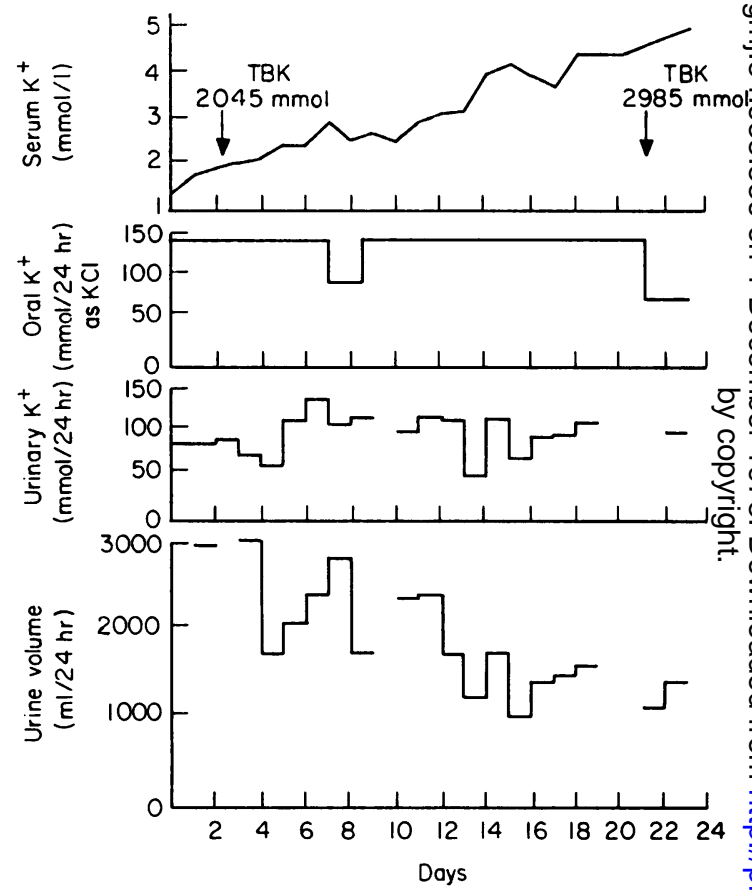

FIG. 1. Showing the change in serum potassium, total body potassium, urinary potassium excretion and urine volume in response to oral potassium therapy.

sodium 238 ; and potassium 89 . The calculated urinary osmolality was $365 \mathrm{mosmol} / \mathrm{l}$. Total body potassium $\mathrm{x}$ (TBK) measured on a total body counter was $\rightarrow$ 2405 mmol (normal range for his weight 2782-3665 mmol). He was treated with oral potassium chloride $N$ only and his TBK rose to $2985 \mathrm{mmol}$. Figure $1_{\mathrm{N}}$ shows the oral potassium intake, changes in plasma ${ }_{N}$ potassium, urinary potassium excretion and urine $\omega$ volume. Plasma sodium, bicarbonate, SGOT and? bilirubin returned to normal during his admission and serum creatinine remained normal. On the fourteenth hospital day, after 12-hr fluid deprivation, $\stackrel{+}{-}$ the urinary osmolality was $500 \mathrm{mosmol} / \mathrm{and}$ did 
not increase following an intramuscular injection of five units of vasopressin. Serum arginine vasopressin (AVP) measured the next day was $3.4 \mathrm{ng} / \mathrm{ml}$ (normal value for his urinary osmolality $0.01-0.1 \mathrm{ng} / \mathrm{ml}$ ) (Thomas and Lee, 1976). He made satisfactory progress and was discharged 3 weeks after admission.

A repeat vasopressin test 6 weeks after discharge revealed an inability to concentrate the urine. A water deprivation test 19 weeks after discharge showed his urinary osmolality to be $810 \mathrm{mosmol} / 1$ after 12-hr fluid withdrawal, and his plasma AVP was normal.

\section{Discussion}

Although carbenoxolone-induced hypokalaemia is well recognized there has been only one well documented report of renal tubular dysfunction occurring in association with carbenoxolone-induced hypokalaemia (Mitchell, 1971).

It has become recognized that hypokalaemia in these patients may be a reflection of total body potassium depletion although there have been no reports of the TBK in patients with severe side effects of therapy (Edmonds and Tomkins, 1975). Measurement of TBK is not generally available but recent work has shown that hypokalaemia of the degree found in this patient reflects gross potassium depletion (Morgan and Swaminathan, personal communication). This suggests that the renal lesion in hypokalaemic nephropathy may be secondary to the intracellular potassium depletion reflected by the hypokalaemia rather than the hypokalaemia itself. Although hypokalaemic nephropathy is well recognized there are few reports in the literature. The lesion in this syndrome is thought to reside in the renal tubular cell (Relman and Schwartz, 1965) and, in the light of the discussion above, may be secondary to intracellular potassium depletion. There was clear evidence of tubular dysfunction in this patient: in spite of profound potassium depletion the urinary potassium level was high, indicating a failure of tubular ionic exchange and necessitating high dosage of potassium supplements. Further evidence of tubular dysfunction is evinced from the failure of concentration of the urine following fluid deprivation in the presence of a high level of endogenous AVP. Tubular function returned between 9 and 22 weeks after presentation.

The rapid onset of urinary symptoms in this patient following administration of the diuretic implies that considerable potassium depletion had occurred on carbenoxolone alone and underlines the need for careful monitoring of patients on this drug.

\section{Acknowledgments}

We thank Dr A. T. R. Axon for permission to report this patient; Professor D. B. Morgan for helpful advice; Dr L. Burkinshaw for TBK measurements; Dr T. Thomas for AVP measurements and Dr I. Watt and the nursing staff of Ward 9 for their assistance.

\section{References}

Edmonds, C.J. \& Tomkins, A.M. (1975) Carbenoxolone effects on body potassium and on the bowel. In: Fourth Symposium on Carbenoxolone (Ed. by Avery-Jones, F. \& Parke, D.V.), p. 129. Butterworths, London.

Mitchell, A.B.S. (1971) Duogastrone-induced hypokalaemic nephropathy and myopathy with myoglobinuria. Pos:graduate Medical Journal, 47, 807.

Relman, A.S. \& Schwartz, W.B. (1956) Nephropathy of potassium depletion: clinical and pathological entity. New England Journal of Medicine, 255, 195.

Thomas, T.H. \& LeE, M.R. (1976) The specificity of antisera for the radio-immunoassay of arginine vasopressin in human plasma and urine during water loading and dehydration. Clinical Science and Molecular Medicine, 51, 525. 